\begin{tabular}{|c|c|}
\hline Title & Characterization and structural investigation of fractal porous silica over an extremely wide scale range of pore size \\
\hline Author(s) & $\begin{array}{l}\text { Ono, Y usuke; Mayama, Hiroyuki; Furó, István; Sagidullin, A lexander I.; Matsushima, Keiichiro; Ura, Haruo; } \\
\text { U chiyama, Tomoyuki; T sujii, Kaoru }\end{array}$ \\
\hline Citation & $\begin{array}{l}\text { Journal of Colloid and Interface Science, 336(1), 215-225 } \\
\text { https://doi.org/10.1016/.jcis.2009.03.087 }\end{array}$ \\
\hline Issue Date & $2009-08-01$ \\
\hline Doc URL & http:/hdl.handle.net/2115/38726 \\
\hline Type & article (author version) \\
\hline File Information & 336-1_p215-225.pdf \\
\hline
\end{tabular}

Instructions for use 


\title{
Characterization and structural investigation of fractal porous-silica over an extremely wide scale range of pore size
}

\author{
Yusuke Ono ${ }^{1}$, Hiroyuki Mayama ${ }^{1, *}$, István Furó ${ }^{2}$, Alexander I. Sagidullin ${ }^{2}$, Keiichiro \\ Matsushima $^{3}$, Haruo Ura ${ }^{3}$, Tomoyuki Uchiyama ${ }^{3}$ and Kaoru Tsujii ${ }^{1,4}$ \\ ${ }^{1}$ Nanotechnology Research Center, Research Institute for Electronic Science, Hokkaido \\ University, Sapporo 001-0021, Japan \\ ${ }^{2}$ Division of Physical Chemistry and Industrial NMR Centre, Department of Chemistry, Royal \\ Institute of Technology, SE-10044 Stockholm, Sweden \\ ${ }^{3}$ Hokkaido Industrial Research Institute, Sapporo 060-0819, Japan \\ ${ }^{4}$ CREST, JST, Kawaguchi, Saitama 338-8570, Japan
}

\begin{abstract}
We have succeeded in creating Menger sponge-like fractal body, i.e., porous-silica samples with Menger sponge-like fractal geometries, by a novel template method utilizing template particles of alkylketene dimer (AKD) and a sol-gel synthesis of tetramethyl orthosilicate (TMOS). We report here the first experimental results on characterization and structural investigations of the fractal porous-silica samples prepared with various conditions such as calcination temperature and packing condition of the template particles. In order to characterize the fractal porous-silica samples, pore volume distribution, porosity and specific surface area were measured over an extremely wide scale from $1 \mathrm{~nm}$ to $100 \mu \mathrm{m}$ by means of mercury porosimetry, ${ }^{1} \mathrm{H}$ NMR cryoporometry, and nitrogen gas adsorption experiments together with direct evaluations of cross-sectional fractal dimension $D_{\mathrm{cs}}$, size limits of $D_{\mathrm{cs}}$. We have found that the pore volume distribution and specific surface area of the fractal porous-silica samples can be discussed in terms of different fractal porous structures at different scale regions.
\end{abstract}

Keywords: Fractal; Pore size distribution; Specific surface area; Hierarchical porous structure;

Sol-gel synthesis; Mercury porosimetry; ${ }^{1} \mathrm{H}$ NMR cryoporometry; Nitrogen gas adsorption

*Corresponding author. Fax:+81 11706 9346. E-mail address: mayama@es.hokudai.ac.jp 


\section{Introduction}

Fractal is a mathematical concept to explain how similar patterns appear at different scales such that the coastline of the Scandinavian Peninsula and the shape of clouds appear [1,2]. In addition, the fractal concept has been applied to interpret many phenomena such as the 1/f-noise in rhythm [3], the correlation of one-dimensional base-sequence in DNA [4], critical phenomena [5], pattern formation of bacteria colony [6], distribution of population in municipalities [7], etc. However, it has been clarified recently that the fractal is also fruitful in material designs. For instance, fractal surface structures emerge super-water repellency [8-14] and super oil-repellency [15]. The super water-repellent fractal structure is spontaneously formed on the surfaces of alkylketene dimer (AKD) and triglycerides waxes [8-10,12-14]. The super-water repellency is caused by the enhancement of the real surface area of waxes arising from the surface roughness. Another example is Menger sponge-like cavities for localization of electromagnetic waves [16]. In this case, the hierarchical porous structure is essential for the localization.

Very recently we have succeeded in creating "fractal body", porous-silica samples with Menger sponge-like fractal geometries, by a novel template method [17]. We utilized the template particles of AKD and a sol-gel synthesis of tetramethyl orthosilicate (TMOS), where AKD is a material of super water-repellent fractal surface and the template particles are corresponding to pores. We stacked the AKD particles having the fractal surface into a vessel 
with suitable compression ratio $\left(r_{\mathrm{c}}\right)$ and made $\mathrm{SiO}_{2}$ replica of the space between the particles by the sol-gel synthesis. Next, the obtained $\mathrm{SiO}_{2}$ including the AKD particles was heated at an appropriate temperature to remove by burning the wax particles from the sol-gel product. We thus created the fractal body possessing the pore size distribution of $50 \mathrm{~nm} \sim 30 \mu \mathrm{m}$. In order to evaluate the fractal geometry, cross-sectional fractal dimension $D_{\mathrm{cs}}$ and its scale range, volume fraction of silica, upper and lower cutoffs of the fractal (self-similar) structure and porosity were observed. Comparing the mathematical models of fractal body, it was concluded that the fractal geometry of the sample depended on the packing condition of the AKD template particles and changed from Menger sponge $(D \sim 2.73)$ for natural packing $\left(r_{\mathrm{c}}=1\right)$ to its modified model $(D \sim$ 2.5 ) for three times compression $\left(r_{\mathrm{c}}=3\right)$. In other words, we found that $D$ can be controlled by the packing condition of template particles. Furthermore, we have determined $D$ of the fractal bodies themselves by an extremely wide scale observation with a combination of ultra-small-angle neutron scattering (USANS), small-angle neutron scattering (SANS) and small-angle X-ray scattering (SAXS) over five orders from $1 \mathrm{~nm}$ to ca. $100 \mu \mathrm{m}$ [18]. The observed $D$ values were close to those of Menger sponge-like structures at the range of $100 \mathrm{~nm}$ $\sim 1 \mu \mathrm{m}$ as predicted in our previous work [17].

Further characterization such as pore size and volume distributions and specific surface area are crucially important from both viewpoints of scientific significance and practical applications of this novel material. Since we found that D was reduced with increasing rc, it is interesting to 
examine the change of the pore size and volume distributions and the specific surface area with $r_{\mathrm{c}}$. Moreover, the calcination temperature $T_{\text {cal }}$ in the sol-gel synthesis of $\mathrm{SiO}_{2}$ affects strongly the small pores arising from a gel network of $\mathrm{SiO}_{2}$. Therefore, the pore characterization with the changes of calcination temperature and $r_{\mathrm{c}}$ in fractal porous-silica should be systematically investigated. In this article, we report the first experimental results on the pore characterization of the fractal porous-silica samples with the changes of $T_{\text {cal }}$ and $r_{\mathrm{c}}$ over an extremely wide scale range from $1 \mathrm{~nm}$ to $100 \mu \mathrm{m}$ by the set of different techniques of SEM observation, mercury porosimetry, ${ }^{1} \mathrm{H}$ NMR cryoporometry [19-22] and nitrogen gas adsorption technique. We will address a correlation between the pore characterization and the fractal geometries.

\section{Experimental section}

\subsection{Sample preparation}

The fractal bodies were prepared as described elsewhere [17]. First, fractal particles were prepared by a spray technique using nitrogen gas (the gas flow rate is $\sim 3$ liter min-1) from a n-hexane (Wako) solution of alkylketene dimer (AKD, supplied by Arakawa Chemical Industries, Ltd.) at ca. $5 \mathrm{wt} \%$, where AKD was preliminarily recrystallized from n-hexane before use. The sprayed particles were kept at room temperature for several days to form fractal surface structure spontaneously. Next, we stacked the particles into a vessel and compressed under compaction ratios $r_{\mathrm{c}}=1,2$, and 3 , where $r_{\mathrm{c}}=1$ means the natural packing of particles, and 
$r_{\mathrm{c}}=2$ and 3 mean that the stacked particles were compressed to the volume ratio of $1 / r_{\mathrm{c}}$. A solution of tetramethyl orthosilicate (TMOS, Shin-Etsu Chemical Co., Ltd.) that was the mixture of TMOS, pure water and methanol at 1: $4: 5$ in molar ratio at $\mathrm{pH}=6.86(\mathrm{pH}$ standard phosphate buffer solution, Wako) was added into remained space between the particles. The samples were left to stand at room temperature for 3 days during the sol-gel reaction process. Then, they were calcined at the calcination temperature $T_{\text {cal }}=500\left(r_{\mathrm{c}}=1\right), 650\left(r_{\mathrm{c}}=1,2\right.$, and 3), $700\left(r_{\mathrm{c}}=1,2\right.$, and 3$), 800\left(r_{\mathrm{c}}=1,2\right.$, and 3$)$, and $900{ }^{\circ} \mathrm{C}\left(r_{\mathrm{c}}=1,2\right.$, and 3$)$ for $2 \mathrm{~h}$ in ambient air to remove the AKD template particles, respectively.

\subsection{Fractal analysis}

We determined the cross-sectional fractal dimension $D_{\mathrm{cs}}$ of the fractal bodies by direct observations of cross sections at different magnifications with scanning electron microscopy (SEM). Sample was crushed into small pieces, and those of the order of $500 \mu \mathrm{m}$-size were used for fabrication by a focused ion beam (FIB) (Hitachi-2100) to obtain clear cross sections. From the cross-sectional SEM images, two-digit images were obtained by image processing and the fractal dimension $D_{\mathrm{cs}}$ was determined by a box-counting method.

\subsection{Pore size distribution, porosity and specific surface area}

Pore size distribution was measured by three different experimental techniques, i.e., mercury porosimetry, ${ }^{1} \mathrm{H}$ NMR cryoporometry [19-22] and nitrogen gas adsorption technique. Usually, the first one covers the pore-size range from ca. $10 \mathrm{~nm}$ to $1 \mathrm{~mm}$, the second one does 
from several $\mathrm{nm}$ to $1 \mu \mathrm{m}$, and the last one from $1 \mathrm{~nm}$ to $50 \mathrm{~nm}$ at most. We adopted these techniques to investigate the pore character over an extremely wide scale. Porosity was calculated from the mercury porosimetry and specific surface area was determined by the nitrogen gas adsorption experiments.

The pore size distribution and the porosity were measured by mercury porosimetry technique using a mercury porosimeter (CE Instruments Porosimeter Pascal 140, 440). Before the experiment, the silica samples were crushed into mm-size grains. The dilatometer containing each sample was degassed under vacuum and then filled up with mercury. The pressurization was up to $200 \mathrm{MPa}$. The pressure $P$ is related with the pore size $r$ as Eq. (1) [23].

$$
r=-4 \gamma \cos \theta / P
$$

where $\gamma$ is the surface tension of mercury, $\theta$ is the contact angle between mercury and silica. Usually, $r$ in mercury porosimetry is calculated under the conditions of $\gamma=484 \mathrm{mN} \mathrm{m}^{-1}, \theta=$ $140^{\circ}$. The schematic representation of the experimental principle is illustrated as the inset of Fig. 5 (a). Here, the pore is assumed to be a cylinder. The pore volume at $r$ was determined from the volume change of mercury in the outside of the fractal porous-silica samples at $P$.

The pore size distribution was also measured by the ${ }^{1} \mathrm{H}$ NMR cryoporometry as follows [19-22]. The principle is the melting temperature depression in small pores. The relation between melting depression $\Delta T_{\mathrm{m}}$ and pore size $r$ is [20] 


$$
\Delta T_{m}=-\frac{2 v \gamma_{s l} T_{m}^{0}}{\Delta H} \cdot \frac{1}{r}
$$

where $\Delta T_{\mathrm{m}}$ is $T_{m}-T_{m}^{0}, T_{m}^{0}$ and $T_{\mathrm{m}}$ are the melting points in bulk medium and in the pore, respectively, $r$ is the diameter of spherical pore, $v$ is the molar volume of medium, $\gamma_{\mathrm{sl}}$ is the interfacial energy between solid and liquid, $\Delta H$ is the latent heat of melting, and $v \gamma_{s l} T_{m}^{0} / \Delta H=56.5 \mathrm{~K} \cdot \mathrm{m}$ for octamethylcyclotetrasiloxane (OMCTS $)\left(v=279.8 \times 10^{-3} \mathrm{~m}^{3} \mathrm{~mol}^{-1}\right.$, $\gamma_{S L}=13.7 \mathrm{~mJ} \mathrm{~m}^{-2}, T_{m}^{0}=290.4 \mathrm{~K}$, and $\Delta H=19.7 \times 10^{3} \mathrm{~J} \mathrm{~mol}^{-1}$ ) [21]. $T_{\mathrm{m}}$ can be detected experimentally because of the huge difference between the transverse relaxation times $T_{2}$ in solid and liquid states, and the pore volume is evaluated from the intensity of the NMR signal (see below). Thus dependence of cumulative pore volume on pore size can be measured by the ${ }^{1} \mathrm{H}$ NMR with temperature change.

The sample prepared at $r_{\mathrm{c}}=1$ and $T_{\text {cal }}=650{ }^{\circ} \mathrm{C}$ was preliminarily crushed into $\sim \mathrm{mm}$ size and the particles with much smaller size than $\sim$ mm were removed. The particles were filled into $5 \mathrm{~mm}$ NMR sample tube. Then the sample was heated at $125{ }^{\circ} \mathrm{C}$ for $15 \mathrm{~h}$ and then vacuum-pumped several hours at room temperature. The mass of sample was $15.0 \mathrm{mg}$ at this stage. Next, $69.1 \mathrm{mg}$ of OMCTS, a probe liquid, was filled into the tube. The ${ }^{1} \mathrm{H}$ NMR cryoporometry was carried out with a Bruker DMX500 spectrometer equipped with a conventional $5 \mathrm{~mm}$ NMR probe with a $90^{\circ}$ pulse length of $4.8 \mu \mathrm{s}$. The sample temperature was controlled by an accuracy of $\pm 0.1 \mathrm{~K}$ by a Bruker BVT2000 temperature regulator. The sample was initially cooled to ca. $160 \mathrm{~K}$ from which the temperature was stepwise increased. The 
waiting time between a temperature step and the start of NMR experiment was at least 5 minutes with a maximum rate of temperature increase of $0.5 \mathrm{~K} \mathrm{~min}^{-1}$. The liquid NMR signal intensity $I(T)$ was recorded by a Car-Purcell-Meiboom-Gill spin-echo-train filter consisting of four $180^{\circ}$ pulses and with a total echo time of $5.36 \mathrm{~ms}$. The applied pulse sequence cancels the solid signal, and one therefore detects the temperature dependence of the liquid NMR intensity. This intensity at a particular temperature shift $\Delta T_{\mathrm{m}}$ corresponds to the total volume of pores with sizes below the volume given by Eq. (2). Hence, the temperature dependence of the liquid NMR intensity can be, via Eq. (2), easily rescaled into the cumulative pore volume distribution.

The pore size distribution and specific surface area were also measured at $-196{ }^{\circ} \mathrm{C}$ by the nitrogen gas adsorption experiments using Autosorb 6AG apparatus (Yuasa Ionics Inc.) of open facility of Creative Research Initiative "Sosei", Hokkaido University. Prior to the measurements, the samples were crushed into mm-size grains and each sample was degassed in vacuo for $2 \mathrm{~h}$ at $120{ }^{\circ} \mathrm{C}$. The specific surface areas were calculated from the Brunauer-Emmett-Teller (BET) equation. Pore size and pore volume were calculated by Dollimore-Heal (DH) method [24] based on the desorption branch of the isotherms.

\section{Results}

Figs. 1 (a) - (c) exhibit typical SEM images of the cross sections and their corresponding two-digit images of the fractal porous silica prepared at $r_{\mathrm{c}}=1$ and $T_{\text {cal }}=650^{\circ} \mathrm{C}$. As shown in the 
two-digit images of Figs. 1 (a) - (c), it is obvious that different sizes and shapes of pores distribute over the real cross sections and similar patterns appear at different magnifications. This presents "self-similarity" on the pore size distribution at least over one decade. From the SEM images of Figs. 1 (a) and (b), the pores are the replicas of the template particles. The long and thin pores observed at higher magnification are the cross sections of wax flakes appeared on the particle surfaces. Fig. 1 (d) is the analytical result of box-counting method for at most 20 two-digit images. The dependence obeys a power law of $N(r) \propto r^{-D c s}$, but two slight inflection points indicated by the arrows are found at ca. $100 \mathrm{~nm}$ and $6 \mu \mathrm{m}$. In the intermediate scale range between $100 \mathrm{~nm}$ and $6 \mu \mathrm{m}, D_{\mathrm{cs}}=1.87$. This means that $100 \mathrm{~nm}$ and $6 \mu \mathrm{m}$ are corresponding to lower and upper cutoffs, where the lower and upper cutoffs were originated from the thickness of a flake and the maximum size of template AKD particles, respectively. On the other hand, $D_{\mathrm{cs}}=2.0$ is realized within the range of below the lower cutoff and above the upper one. Figs. 2 and 3 also show the cross-sectional SEM images and analytical results for fractal porous silicas prepared at $r_{\mathrm{c}}=2,3$ and $T_{\text {cal }}=650{ }^{\circ} \mathrm{C}$, respectively. Smaller pores are apparently observed in the two-digit images and $D_{\mathrm{cs}}=1.87$ and 1.84 were obtained for the samples $r_{\mathrm{c}}=2,3$, respectively.

Fig. 4 (a) shows the dependence of $D_{\text {cs }}$ at different $r_{\mathrm{c}}$ on $T_{\text {cal. }}$. In contrast to our previous results [17], a clear dependence of $D_{\mathrm{cs}}$ on $r_{\mathrm{c}}$ could not be observed at the same $T_{\mathrm{cal}}$. On the other hand, $D_{\text {cs }}$ seems to decrease with increasing $T_{\text {cal }}$. The dependences of upper cutoff $L$ and lower 
one $l$ on $T_{\text {cal }}$ are shown in Fig. 4 (b). They show clear dependences on $r_{\mathrm{c}}$, which are in good agreement with the findings in our previous work [17], but not dependent on $T_{\text {cal }}$. We will discuss this point later from the viewpoint of the geometry of porous silica.

Fig. 5 shows the pore volume distributions and the cumulative ones obtained by mercury porosimetry in fractal porous silica samples $\left(r_{\mathrm{c}}=1,2\right.$, and 3$)$ and ordinary silica obtained at $T_{\text {cal }}$ $=650{ }^{\circ} \mathrm{C}$, where the ordinary silica was prepared under same procedure using no template AKD particles. The fractal porous-silica samples exhibited wide size distributions over three decades between ca. $10 \mathrm{~nm}$ and ca. $10 \mu \mathrm{m}$ as shown in Figs. 5 (a) - (c). The pore size distributions in the samples at $r_{\mathrm{c}}=2$ and 3 show similar characteristics to those at $r_{\mathrm{c}}=1$ in the range between 10 $\mathrm{nm}$ and $1 \mu \mathrm{m}$, but the distributions at larger sizes were somewhat different. The maximum size increases from $5 \mu \mathrm{m}$ to $200 \mu \mathrm{m}$ with increasing $r_{\mathrm{c}}$. This is probably related to the collapse of the AKD template particles upon compression. Furthermore, the cumulative pore volume of the samples systematically increases by more than four times from ca. $1200 \mathrm{~mm}^{3} \mathrm{~g}^{-1}$ to ca. 5200 $\mathrm{mm}^{3} \mathrm{~g}^{-1}$ with increasing $r_{\mathrm{c}}$. Contrary to the fractal porous-silica, the normal $\mathrm{SiO}_{2}$ glass which was prepared by the same procedures but without AKD particles had a very small cumulative pore volume on the order of $3.8 \%$ of that obtained at $r_{\mathrm{c}}=1$. The quite small pores of the $\mathrm{SiO}_{2}$ are formed as silica-gel networks during the sol-gel reaction process, and contribute little to the total pore volume in the silica material. The shapes of the distributions of relative pore volume were similar even at different $T_{\text {cal }}$ (data not shown here). This probably indicates that the fractal 
porous silica sample was shrunk homogeneously with increasing $T_{\text {cal }}$.

Fig. 6 (a) shows the dependence of the total pore volume of the samples $V_{\mathrm{Hg}}$ at each $r_{\mathrm{c}}$ and of normal $\mathrm{SiO}_{2}$ on $T_{\text {cal }}$. The $V_{\mathrm{Hg}}$ depends strongly on $r_{\mathrm{c}}$, but weakly on $T_{\text {cal. }}$. The dependence of porosity on $T_{\text {cal }}$ is shown in Fig. 6 (b). Although $D_{\text {cs }}$ did not show clear dependence on $r_{\mathrm{c}}$ as described in Fig. 4, the porosity was apparently decreased with increasing $r_{\mathrm{c}}$ and $T_{\text {cal }}$. Here, the values of porosity were calculated from the mass of samples, the density of material $(\rho=2.4 \mathrm{~g}$ $\mathrm{cm}^{-3}$ for $\mathrm{SiO}_{2}$ ), and the volume of mercury inserted into the samples. The porosity of the sample $p(\%)$ is then written as

$$
p=\frac{V_{H g}}{V_{H g}+V_{\text {material }}} \times 100
$$

where $V_{\text {material }}$ is the net volume of materials calculated from density of material $\left(\mathrm{SiO}_{2}\right)$ and mass of fractal body.

Fig. 7 (a) shows the dependence of cumulative pore volume distributions on $r$ of the fractal porous silica at $r_{\mathrm{c}}=1$ and $T_{\text {cal }}=650{ }^{\circ} \mathrm{C}$ obtained by the ${ }^{1} \mathrm{H}$ NMR cryoporometry. Fig. 7 (b) shows two peaks in the pore size distribution. One is in the range of $r>100 \mathrm{~nm}$ and the other is at $r<10 \mathrm{~nm}$. The former and the latter would reflect a hierarchical porous structure formed with the AKD templates and the mesh size of a gel network of $\mathrm{SiO}_{2}$ in the sol-gel synthesis, respectively.

Figs. 8 (a) - (c) show the dependences of cumulative pore volume on pore size for the samples at $r_{\mathrm{c}}=1,2$, and 3, respectively. With increasing $T_{\text {cal }}$ at all $r_{\mathrm{c}}$, the cumulative pore 
volume $V$ is systematically decreased. In particular, the pore volume of relatively smaller pores seems to be collapsed because the increase of $V$ at $r<4 \mathrm{~nm}$ was suppressed with increasing $T_{\text {cal }}$. The collapse is reflected in the decrease of specific surface area $S$ as shown in Fig. 8 (d) in which the specific surface areas are apparently decreased with an increase of $T_{\text {cal }}$. Contrary to these, no significant difference in $S$ between the porous-silica samples with different $r_{\mathrm{c}}$ and between the porous silica samples and normal $\mathrm{SiO}_{2}$ was observed, and indicating that their porous structures in very smaller scale range were similar.

Fig. 9 shows the pore size distribution over the extremely wide scale range from $1 \mathrm{~nm}$ to $10 \mu \mathrm{m}$ obtained from the mercury porosimetry, the ${ }^{1} \mathrm{H}$ NMR cryoporometry and the nitrogen gas adsorption experiments. Two pore distributions are clearly indicated at $r=1 \sim 10 \mathrm{~nm}$ and 100 $\mathrm{nm} \sim 10 \mu \mathrm{m}$, respectively. In particular, the sharp distribution having a peak at $r=4 \mathrm{~nm}$, which was evaluated from the nitrogen gas adsorption experiments, was clearly shown.

Consequently, the experimental results were summarized as follows.

(1) From the SEM observations of the cross sections (observed scale range; $10 \mathrm{~nm} \sim 100 \mu \mathrm{m}$. Fig. 4)

The $D_{\mathrm{cs}}$ does not depend clearly either on $T_{\text {cal }}$ or on $r_{\mathrm{c}}$. The upper and lower cutoffs did not show the $T_{\text {cal }}$ dependence, but the clear dependence on $r_{\mathrm{c}}$.

(2) From the mercury porosimetry (observed scale range; $10 \mathrm{~nm} \sim 100 \mu \mathrm{m}$. Figs. 5 and 6 )

There are two significant and distinct regions in the pore-size distributions. In the smaller 
scale region $(10 \mathrm{~nm} \sim 1 \mu \mathrm{m})$, the broad pore size distributions in fractal porous-silica samples were observed. The distributions were similar independently on both $r_{\mathrm{c}}$ and $T_{\text {cal }}$. On the other hand, in the larger scale region $(1 \mu \mathrm{m} \sim 100 \mu \mathrm{m})$, the distributions which would result from the AKD template particles were somewhat different in the samples at different $r_{\mathrm{c}}$. The $V_{\mathrm{Hg}}$ and the porosity were decreased with elevating $T_{\text {cal }}$. The homogeneous shrinkage of the fractal porous-silica samples with elevating $T_{\text {cal }}$ were found by the finding that the pore size distributions of the fractal porous-silica samples at same $r_{\mathrm{c}}$ were similar even at different $T_{\text {cal }}$. The pore size of normal $\mathrm{SiO}_{2}$ was in a sharp distribution around $10 \mathrm{~nm}$ in Fig. 5 (d). This distribution was much different from that of the fractal bodies. In addition, the $V_{\mathrm{Hg}}$ was much smaller than that of the fractal bodies.

(3) From the ${ }^{1} \mathrm{H}$ NMR cryoporometry (observed scale range; $10 \mathrm{~nm} \sim 1 \mu \mathrm{m}$. Fig. 7).

Two pore-size distributions in a typical fractal body $\left(r_{\mathrm{c}}=1\right.$ and $\left.T_{\text {cal }}=650{ }^{\circ} \mathrm{C}\right)$ exist at $r>$ $100 \mathrm{~nm}$ and $r<10 \mathrm{~nm}$, respectively. The pore size ranges of the former and latter were in good agreement with those obtained by the mercury porosimetry (Fig. 5) and the nitrogen gas adsorption experiments (see Fig. 9), respectively.

(4) From the nitrogen gas adsorption technique (observed scale range; $2 \mathrm{~nm} \sim 20 \mathrm{~nm}$. Fig. 8).

The cumulative pore volume $V$ and the specific surface area $S$ of the fractal bodies and the normal $\mathrm{SiO}_{2}$ were evaluated. The $V$ and $S$ of the fractal bodies were decreased with increasing $T_{\text {cal }}$. The $S$ of the fractal bodies was similar to that of the normal $\mathrm{SiO}_{2}$. The sharp pore size 
distribution around $4 \mathrm{~nm}$ was observed.

\section{Discussion}

\subsection{Two kinds of pores and their contribution to the pore-volume and specific surface area}

There are two kinds of pores in the fractal porous-silica samples as shown in Fig. 9. One is originated from the AKD template particles, and has the pore-size distribution of about 100 $\mathrm{nm}-10 \mu \mathrm{m}$. The other is intrinsic $4 \mathrm{~nm}$-pores of silica, and is formed during the sol-gel reaction process. The fractal (self-similar) structure is maintained in the size-distribution of the larger pores.

The contribution of these two kinds of pores to the characteristic properties of the silica material is quite discriminatory. The larger fractal pores contribute much to the pore-volume as seen in Figures 5 and 7, and do not at all to the specific surface area. The specific surface area of the fractal porous-silica is almost the same as that of normal silica as shown in Figure 8 (d). The intrinsic smaller pores of silica around $4 \mathrm{~nm}$ in size contribute solely to the specific surface area. Regretfully, the fractal nature of the porous silica created in this study does not seem to lead the larger surface area.

4.2 Porous structure and fractal geometry from $100 \mathrm{~nm}$ to $10 \mu \mathrm{m}$ in the porous-silica made at $r_{c}=1$ and $T_{c a l}=650^{\circ} \mathrm{C}$

As mentioned before, our porous-silica samples possess two kinds of pores, i.e., $4 \mathrm{~nm}$ 
intrinsic pores and the fractal ones with the size distribution from $100 \mathrm{~nm}$ to $10 \mu \mathrm{m}$. We may be able to construct the structural model taking the above facts into account. Let us discuss first the fractal geometry of the porous-silica based on $D_{\mathrm{cs}}$ and porosity. On the other words, we attempt to translate from $D_{\mathrm{cs}}$ in two-dimensional cuts to the mass fractal dimension $D$ in three-dimensional matrices. We adopt the samples prepared under $r_{\mathrm{c}}=1$, and $650{ }^{\circ} \mathrm{C}$ first, since they can be assumed to be a Menger sponge as following reasons. The first reason is that the obtained $D_{\mathrm{cs}}$ is close to that of Menger sponge. Menger sponge is created by identical 20 cubes as shown in Fig. 10 (a) (the first generation). Next, the size of the unit cube is reduced with the size reduction ratio $r_{\text {size }}=1 / 3$, and the second generation hollow cube is created by the identical 20 cubes as shown in Fig. 10 (b). The model in Fig. 10 (c), the 3rd generation, is created by 1/3 size reduction of the model in Fig. 10 (b). This procedure is repeated infinitely. The fractal dimension of a cross section $D_{\mathrm{cs}}$ of Menger sponge is

$$
D_{c s}=-\log 8 / \log (1 / 3)=1.89
$$

Now, the obtained $D_{\mathrm{cs}}$ was 1.87 , which is close to that of Menger sponge.

The fractal geometry should be comprehensively considered from $D_{\mathrm{cs}}, l, L$ and $p$. These parameters are closely related each other as shown below. First, it is easy to understand that $p$ depends on the number of the generations in fractal structure. The relation between the porosity $p$ and the volume reduction ratio of Menger sponge is

$$
p=1-(20 / 27)^{n}
$$


where $n$ is the generation number of hierarchical structure and the factor 20/27 is the volume reduction ratio of Menger sponge. If one can estimate $n$, then the geometry can compare with the model. It can be obtained from the relation between the pore size reduction ratio and the cutoffs [17].

$$
(1 / 3)^{n-1}=l / L
$$

Thus $n$ can be evaluated from the scale range in which $D_{\mathrm{cs}}$ is maintained. Based on Eqs. (5) and (6), we can discuss the geometry of the sample. From Eq. (6), $n \sim 5$ for $L=6 \mu \mathrm{m}$ and $l=100$ $\mathrm{nm}$. Therefore, the theoretical porosity $p$ is evaluated to be $78 \%$ from Eq. (5). The actual porosity obtained from the mercury porosimetry ( $75 \%$ as shown in Fig. 6) is in good agreement with the theoretical value. Thus, it can be concluded that the fractal geometry of the sample is close to Menger sponge between $100 \mathrm{~nm}$ and $6 \mu \mathrm{m}$.

We can further discuss the geometry from the cumulative pore volume along the following scenario. If the pore size at the first stage is $L$ (the size of unit cube is $3 L$ ), then the pore volume generated is $7 L^{3}$. At next stage, the pore volume is $7(L / 3)^{3} \times 20=7 L^{3}(20 / 27)$, where the factor $1 / 3$ is the size reduction ratio to create a Menger sponge. Therefore, the pore volume of the pores at the $n$-th generation $v_{\mathrm{n}}$ is

$$
v_{n}=7 L^{3}\left(\frac{20}{27}\right)^{n-1}=7 \times 20^{n-1} l_{n}^{3},
$$

where the factor $20 / 27$ is the volume reduction ratio and $l_{n}=L / 3^{n-1} \cdot v_{n}$ decreases with the 
development of generation number. The total pore volume $V_{n}$ in a Menger sponge from the first to the $n$-th generation is the sum of series of $v_{n}$

$$
V_{n}=\sum_{n=1}^{n} v_{n}=27 L^{3}\left[1-\left(\frac{20}{27}\right)^{n}\right] .
$$

From Eq. (8), it is obvious that if $n \rightarrow \infty$ then the pore volume reaches to the volume of whole cube $(3 L)^{3}$, i.e., the net volume of mass becomes zero. Eq. (8) is important to evaluate cumulative pore volume $V$ which corresponds to the total pore volume by unit mass. The $V$ of the hierarchical structure from the first generation to the $n$-th generation can be described as

$$
V=\frac{V_{n}}{(3 L)^{3} f_{n} \rho},
$$

where $\rho$ is density of material $\left(2.4 \mathrm{~g} \mathrm{~cm}^{-3}\right.$ for $\left.\mathrm{SiO}_{2}\right)$ and $f_{n}$ is the volume fraction of Menger sponge at the $n$-th generation,

$$
f_{n}=(20 / 27)^{n}
$$

Eqs. (7) - (10) provide us an approach to discuss the fractal geometry of the sample in comparison with mathematical model on the basis of pore volume distribution. Here, to make the calculations of $v_{n}$ and $V$ simple, the shapes of pores were approximated to be cubes.

Fig. 11 shows a comparison with the experimental and theoretical results assuming $L=$ $1.3 \mu \mathrm{m}$ on the dependences of cumulative pore volume and pore volume on pore size using Eqs. (7) - (10). The theoretical results are indicated by the dashed lines, while the solid line and histogram are experimental results in Fig. 5 (a), where the histogram is redrawn from the 
histogram in Fig. 5 (a) to compare with the hierarchical structure (size reduction ratio $=1 / 3$ ).

The redrawn histogram has a peak at ca. $1 \mu \mathrm{m}$ and long tail with size reduction over two decades due to the hierarchical structure. Theoretical results in both pore volume and cumulative one are in fairly good agreement with the experimental data. The upward deviation in the theoretical cumulative volume in the smaller pore region is reasonable, since there is no pore in fractal nature below $100 \mathrm{~nm}$. The best-fit $L$ value was $1.3 \mu \mathrm{m}$ which did not agree well with the upper cutoff $(6 \mu \mathrm{m})$ in Fig. 1. This discrepancy might be originated from the existence of some bottle-neck type pores for which the mercury porosimetry gives smaller size than that of the inner pores of bottles. Thus, the $D$ of the sample is estimated to be close to 2.73 .

\subsection{Porous structures and fractal geometries from $100 \mathrm{~nm}$ to $10 \mu \mathrm{m}$ in the porous-silicas}

prepared at $r_{c}=2,3$ and $T_{\text {cal }}=650{ }^{\circ} \mathrm{C}$

Let us discuss the porous structures and fractal geometries of the porous-silica samples prepared at $r_{\mathrm{c}}=2,3$ and $T_{\mathrm{cal}}=650{ }^{\circ} \mathrm{C}$ on the basis of the experimental results of the cumulative pore volume.

First, let us consider the fractal geometry of the sample of $r_{\mathrm{c}}=2$ and $T_{\text {cal }}=650{ }^{\circ} \mathrm{C}$. The obtained values of $D_{\mathrm{cs}}=1.87, l \sim 0.2 \mu \mathrm{m}, L \sim 6 \mu \mathrm{m}$ and $p=89 \%$. Although $\quad D_{\mathrm{cs}}$ is close to that of Menger sponge, it is very difficult to explain $p=89 \%$ by Eqs. (5) and (6). On the other hand, the values of $l, L$ and $p$ are close to these obtained in our previous experiment $\left(D_{\mathrm{cs}}=1.84\right.$, $l=0.05 \mu \mathrm{m}, L=2 \mu \mathrm{m}$ and $p=92 \%$ ). As a result, it can be concluded that $D_{\text {cs }}$ obtained here 
would be overestimated because of the experimental errors. The fractal geometry should be comprehensively considered on the basis of $D_{\mathrm{cs}}, l, L$ and $p$. Fig. 12 (a) illustrates a candidate of the sponge model $\left(D_{\mathrm{cs}}=-\log 13 / \log (1 / 4)=1.85\right.$ and $\left.D=-\log 39 / \log (1 / 4)=2.64\right)$. Let us discuss the fractal geometry based on $p$ of the model. The relation between $p$ and the volume reduction ratio of this model is

$$
p=1-(39 / 64)^{n}
$$

From this relation, $n$ is estimated to be ca. 5 since $p=90 \%$. On the other hand, the relation between $l, L$ and $n$ is

$$
(1 / 4)^{n-1}=l / L
$$

where the factor $1 / 4$ is the size reduction ratio of this model. From this relation, $n$ is evaluated to be ca. 4. It is understood that the difference of $n$ obitained by Eqs. (11) and (12) would be originated from the experimental errors.

The validity of the model can be discussed in terms of cumulative pore volume. The pore volume of the $n$-th generation $v_{n}$ in this model is

$$
v_{n}=25\left(\frac{L}{2}\right)^{3}\left(\frac{39}{64}\right)^{n-1}
$$

The total pore volume $V_{n}$ from the first to the $n$-th generation is

$$
V_{n}=64\left(\frac{L}{2}\right)^{3}\left[1-\left(\frac{39}{64}\right)^{n}\right]
$$

The total pore volume by unit mass $V$ is 


$$
V=\frac{V_{n}}{(2 L)^{3} f_{n} \rho}
$$

where $f_{n}=(39 / 64)^{n}$. Fig. 12 (b) shows a comparison with the experimental and theoretical results assuming $L=2 \mu \mathrm{m}$ on the dependences of cumulative pore volume and pore volume on pore size in Eqs. (13) - (15). The cumulative pore volume in the size range from $2 \mu \mathrm{m}$ to 0.125 $\mu \mathrm{m}$ in the experimental result can be reproduced by the sponge model as shown in Fig. 12 (a). Thus, $D$ of this sample is estimated to be close to 2.64 .

Next, let us briefly discuss the fractal geometry of the sample at $r_{\mathrm{c}}=3$ and $T_{\text {cal }}=650{ }^{\circ} \mathrm{C}$. The obtained values of $D_{\mathrm{cs}}, l, L$ and $p$ are $1.84,0.2 \mu \mathrm{m}, 7 \mu \mathrm{m}$ and $93 \%$, respectively. Here, we assume the sponge model as shown in Fig. 13 (a) $\left(D_{\mathrm{cs}}=-\log 12 / \log (1 / 4)=1.79, D=-\log \right.$ $32 / \log (1 / 4)=2.5)$. The relation between $p$ and the volume reduction ratio of this model is

$$
p=1-(32 / 64)^{n}
$$

From this relation, $n$ is estimated to be ca. 4 since $p=92.5 \%$. On the other hand, the relation between $l, L$ and $n$ is

$$
(1 / 4)^{n-1}=l / L
$$

From this relation, $n$ is evaluated to be ca. 4. The values of $n$ obtained from Eqs. (16) and (17) are consisted each other.

The validity of the model can be also discussed in terms of cumulative pore volume. The $v_{n}$ in this model is 


$$
v_{n}=32\left(\frac{L}{2}\right)^{3}\left(\frac{32}{64}\right)^{n-1}
$$

The $V_{n}$ from the first to the $n$-th generation is

$$
V_{n}=64\left(\frac{L}{2}\right)^{3}\left[1-\left(\frac{32}{64}\right)^{n}\right]
$$

The total pore volume by unit mass $V$ is

$$
V=\frac{V_{n}}{(2 L)^{3} f_{n} \rho}
$$

where $f_{n}=(32 / 64)^{n}$. Fig. 13 (b) shows a comparison with the experimental and theoretical results assuming $L=3 \mu \mathrm{m}$ on the dependences of cumulative pore volume and pore volume on pore size in Eqs. (18) - (20). The cumulative pore volume below $3 \mu \mathrm{m}$ in the experimental result can be reproduced very well by the sponge model as shown in Fig. 13 (a). Thus, $D$ of this sample is estimated to be close to 2.5 .

In these two sections, we discussed the mass fractal dimension $D$ of the samples on the basis of the experimental results $\left(D_{\mathrm{cs}}, l, L\right.$ and $\left.p\right)$ and the fractal sponge models. On the other hand, we have been carrying out the direct evaluation of $D$ of fractal porous silica samples by scattering studies such as USANS, SANS and SAXS [18]. Therefore, it would be fruitful to briefly address the comparison between the scattering results and the present results here. In the scattering experiments on porous samples, two kinds of fractal dimension can be observed, surface fractal dimension $D_{\mathrm{s}}$ and mass fractal dimension $D$, which emerge as the dependences of scattering intensity $I(q)$ on wave number $q(q=2 \pi / r, r$ : pore size $)$, can be observed, where $I(q)$ 
$\sim q^{-(6-D s)}$ and $I(q) \sim q^{-D}[27,29,30]$. Roughly speaking, the former and latter dependences are observed in the scale ranges below and above the smallest pore size, respectively. On the other words, the air-solid interface over a whole pore network structure is detected in the $q$-range corresponding to the larger size range than the smallest pore size, while the air-solid interface of pores, i.e., a surface roughness, is detected in the q-range corresponding to the smaller size range than the smallest pore size. Actually, the dependence of $I(q)$ changes at several hundred nanometer in our previous study [18], which corresponds to an average of the size of a surface structure of pores (several tens nanometer $\times$ several micrometer $\times$ several micrometer $)$ in reciprocal space. In the scattering studies on the samples at $r_{\mathrm{c}}=1,3$ and $T_{\text {cal }}=650{ }^{\circ} \mathrm{C}$ [18], we found that the values of $D$ in the range of $q \leq 0.01 \mathrm{~nm}^{-1}$ were evaluated to be 2.67 and 2.29, respectively. The obtained values are somewhat smaller than the estimated values $(D \sim 2.73$ and 2.5 , respectively) [17]. Now, further scattering experiments on fractal porous-silica samples by USANS, SANS and SAXS are in progress. Gas adsorption experiment using various gas molecules with different sizes such as nitrogen and butane is suitable to evaluate $D_{\mathrm{s}}$ and $D$ in the scale range smaller than ca $.10 \mathrm{~nm}$ in pore size [31, 32], but not suitable in more larger scale. In the box-counting method, the surface roughness is observed as the contour of pores, but it is not directly reflected in $D_{\text {cs. }}$.

\subsection{Porous structure of the intrinsic pores of $4 \mathrm{~nm}$}

Let us move here to the discussion on the geometry of the intrinsic pores of $4 \mathrm{~nm}$ on the 
basis of the obtained specific surface area $S$. To discuss the relation between $S$ and the pore structure briefly, we consider a rough scheme that there is a spherical pore with diameter $d$ in a cube with size $d$. Since the surface area of a pore $s=4 \pi(d / 2)^{2}$ in the volume $d^{3}$ and the porosity $p=\left(4 \pi(d / 2)^{3} / 3 d^{3}\right)$, then the specific surface area $S$ is

$$
S=\frac{s}{\rho(1-p) d^{3}}=\frac{\pi}{0.476 \rho d} \propto 1 / d
$$

For $d=4 \mathrm{~nm}, S=688 \mathrm{~m}^{2} \mathrm{~g}^{-1}$. Since the obtained $S$ is $\sim 500 \mathrm{~m}^{2} \mathrm{~g}^{-1}$ at $T_{\text {cal }}=650{ }^{\circ} \mathrm{C}$, the distance between the centers of the pores is ideally $4.4 \mathrm{~nm}$ if the $4 \mathrm{~nm}$-pores homogeneously exist in $\mathrm{SiO}_{2}$. Since $S \propto 1 / d$, the contribution of larger pores of the order of $100 \mathrm{~nm}$ and more to $S$ can be neglected. According to the rough estimation, the obtained $S$ is explained by the existence of the $4 \mathrm{~nm}$-size pores, and not the hierarchical fractal structure. Since it is well-known that usual specific surface area of silica gel is in the order of $\sim 500 \mathrm{~m}^{2} \mathrm{~g}^{-1}[25]$, the obtained $S$ is in good agreement with that of usual $\mathrm{SiO}_{2}$.

\subsection{Effects of $T_{\mathrm{cal}}$ and $r_{\mathrm{c}}$ for porous structure and fractal geometry}

We have discussed so far on the porous structures and fractal geometry of the silica samples made at $r_{\mathrm{c}}=1,2,3$ and $T_{\text {cal }}=650{ }^{\circ} \mathrm{C}$. The fractal structures of the silica materials may be changed when we change the preparation conditions of $r_{\mathrm{c}}$ and $T_{\text {cal }}$. Let us discuss first on the effects of $T_{\text {cal }}$ from the porosity, pore size distribution, $V$ and $S$. Roughly speaking, $D_{\text {cs }}$ (Fig. 4 (a)), $V$ (Fig. 6 (a)), the porosity (Fig. 6 (b)) and $S$ (Fig. 8 (d)) decreased with increasing $T_{\text {cal. }}$ All these results are originated from the shrinkage of the silica material and the collapse of small 
pores by the elevated temperature during the calcination process. For example, the decrease of $D_{\text {cs }}$ could be explained as follows. Figs. 14 (a) and (b) illustrates the Sierpinsky carpet $\left(D_{\mathrm{cs}}=\right.$ $1.89)$ and the modified one $\left(D_{\mathrm{cs}}=1.79\right)$. These structural changes could be possible by the shrinkage of the silica material and the collapse of small pores.

Next, the effect of $r_{\mathrm{c}}$ on the porous structures will be discussed. In our previous study, the fractal geometry in the range from $50 \mathrm{~nm}$ to $20 \mu \mathrm{m}$ was affected by $r_{\mathrm{c}}$ because the porous structure (porosity, pore size, etc.) was changed by the compression of the AKD particles corresponding to the pores in fractal silica samples [17]. For example, the $D_{\mathrm{cs}}$ (porosity) was systematically decreased from $1.89\left(r_{\mathrm{c}}=1, p=85 \%, T_{\text {cal }}=500{ }^{\circ} \mathrm{C}\right), 1.84\left(r_{\mathrm{c}}=2, p=92 \%, T_{\text {cal }}\right.$ $\left.=650{ }^{\circ} \mathrm{C}\right)$ to $1.80\left(r_{\mathrm{c}}=3, p=96 \%, T_{\text {cal }}=650{ }^{\circ} \mathrm{C}\right)$ with increasing $r_{\mathrm{c}}$ in our previous study [17]. Concerning the porosity, the results of the present work (the porosity at $r_{\mathrm{c}}=2,3$ and $T_{\text {cal }}=650$ ${ }^{\circ} \mathrm{C}$ were 89 and $93 \%$, respectively) were in good agreement with those of the previous one. The $D_{\text {cs }}$ values, however, have no systematic change with $r_{\mathrm{c}}$ in the present work. It would be due to the anisotropy of the AKD template particles when compressed in a vessel. The porous-silica samples were, of course, the replica of these anisotropic structures. The $D_{\mathrm{cs}}$ values of the silica samples could be different when measured for the surfaces cut by different directions. This may be why the $D_{\text {cs }}$ values are scattered, and the systematic change was not observed.

It is well-known that porous materials such as active carbon, Vycor glass and aerogels 
have fractal geometry with limited size-scale over only one decade or less around $10 \mathrm{~nm}$ [26-29].

In contrast to these materials, our fractal porous-silica created by the designed template method shows the fractality in the multi-scale from $100 \mathrm{~nm}$ to ca. $10 \mu \mathrm{m}$. In order to create fractal porous-silica with ideal fractal geometries, experimental design for the porous structure at $r=$ $10 \sim 100 \mathrm{~nm}$ is necessary. If it would be accomplished, then fractal geometry would be maintained over the extremely wide scale from $\mathrm{nm}$ to $\mu \mathrm{m}$ and huge $S$ and almost zero mass would be realized. It would be quite important not only in application, but also in the experiments of phase transition phenomena in the fractal system [33,34]. Further studies would serve us not only possibilities in material design, but also in fundamental physics.

\section{Conclusion}

In summary, we have systematically evaluated $D_{\mathrm{cs}}, L, l$, porosity, $V$, pore size distribution and $S$ of fractal porous-silica samples prepared at different $r_{\mathrm{c}}$ and $T_{\text {cal }}$ over four decades by the combination of SEM observations, mercury porosimetry, the ${ }^{1} \mathrm{H}$ NMR cryoporometry and the nitrogen gas adsorption experiments, and also have discussed the porous structures in fractal porous-silica at different scale regions. We found that there were two different porous structures at different scales in typical samples $\left(r_{\mathrm{c}}=1,2,3\right.$ and $\left.T_{\text {cal }}=650^{\circ} \mathrm{C}\right)$; Menger sponge-like porous structure in the range of ca. $100 \mathrm{~nm}$ - ca. $10 \mu \mathrm{m}$, and the sponge structure with $4 \mathrm{~nm}$-size pores. We also found that $T_{\text {cal }}$ affects strongly the porous structure below $10 \mathrm{~nm}$. The $r_{\mathrm{c}}$ changes mainly 
the porosity, and does not show the clear effect on the fractal dimension.

\section{Acknowledgement}

This work was supported by a Grant-in-Aid for Young Scientists (B) (Grant No. 18710089)

from Ministry of Education, Culture, Sports, Science and Technology, Japan (MEXT) to one author (H.M.). The AKD sample is a kind gift from Arakawa Chemical Industries, Ltd. 


\section{References}

1. B. B. Mandelbrot, The Fractal Geometry of Nature (Freeman, New York, 1977).

2. S. Lovejoy, Science 216 (1982) 185.

3. H. -O. Peitgen \& D. Saupe, The Science of Fractal Images, Springer-Verlag (1988).

4. R. F. Voss, Phys. Rev. Lett. 68 (1992) 3805.

5. H. E. Stanely, Introduction to phase transitions and critical phenomena (Clarendon Press, Oxford, 1971).

6. H. Shimada, T. Ikeda, J. Wakita, H. Itoh, F. Hiramatsu, M. Nakatsuchi, Y. Yamazaki, T. Matsuyama, and M. Matsushita, J. Phys. Soc. Jpn. 73 (2004) 1082.

7. Y. Sasaki, H. Kunitake, N. Kobayashi, and M. Matsushita, J. Phys. Soc. Jpn. 76 (2007), 074801.

8. T. Onda, S.Shibuichi, N. Satoh, and K. Tsujii, Langumir 12 (1996) 2125.

9. S. Shibuichi, T. Onda, N. Satoh, and K. Tsujii, J. Chem. Phys. 100 (1996) 512.

10. S. Shibuichi, T. Yamamoto, T. Onda, and K. Tsujii, J. Colloid, Interface Sci. 208 (1998) 287.

11. K. Kurogi, H. Yan, H. Mayama, and K. Tsujii, J. Colloid Interface Sci. 312 (2007) 156.

12. T. Minami, H. Mayama, S. Nakamura, S. Yokojima, J. -W. Shen, and K. Tsujii, Soft Matter 4 (2008) 140.

13. W. Fang, H. Mayama, and K. Tsujii, J. Phys. Chem. B 111 (2007) 564.

14. W. Fang, H. Mayama, and K. Tsujii, Colloid Sci. A 316 (2008) 258. 
15. K. Tsujii, T. Yamamoto, T. Onda, and S. Satoh, Angew. Chem. Int. Ed. 36 (1997) 1011.

16. M. W. Takeda, S. Kirihara, Y. Miyamoto, K. Sakoda, and K. Honda, Phys. Rev.Lett. 92 (2004) 093902.

17. H. Mayama and K. Tsujii, J. Chem. Phys. 125 (2006) 124706.

18. D. Yamaguchi, H. Mayama, S. Koizumi, K. Tsujii, and T. Hashimoto, Eur. Phys. J. B 63 (2008) 153.

19. J. H. Strange and M. Rahman, Phys. Rev. Lett. 71 (1993) 3589.

20. O. Petrov, I. Furó, Phys. Rev. E 73 (2006) 011608.

21. D. Vargas-Florencia, O. V. Petrov, I. Furó, J. Colloid Interface Sci. 305 (2007) 280.

22. O. V. Petrov, D. Vargas-Florencia, I. Furó, J. Phys. Chem. B 111 (2007) 1574.

23. H. L. Litter and L. C. Drake, Ind. Eng. Chem. Anal. Ed. 17, 782 (1945).; L. C. Drake, Ind. Eng. Chem. 41 (1949) 780.

24. D. Dollimore and G. R. Heal, J. Appl. Chem. 14 (1964) 309.

25. K. C. Chen, T. Tsuchiya, and J. D. Machenzie, J. Non-Cryst. Solids 81 (1986) 227.

26. A. Bóta, K. Lászlo, L. G. Nagy and T. Copitzky, Langmuir 13 (1997) 6502.

27. D. W. Schaefer and K. D. Keeper, Phys. Rev. Lett. 56 (1986) 2199.

28. D. Rojansky, D. Huppert, H. D. Dacai, P. W. Schimitt, D. Farin, D. Seri-Levy and D. Avnir, Phys. Rev. Lett. 56 (1989) 2505.

29. P. W. Schmidt, A. Höhr, H. -B. Naumann, H. Kaiser, D. Avnir and J. S. Lin, J. Chem. Phys. 
90 (1989) 5016.

30. C. -H. Sun, F. L. Ying, C. Liu and H. -M. Cheng, Phys. Rev. B 69 (2004) 033404.

31. M. J. Watt-Smith, .K. J. Edler and S. P. Rigby, Langmuir 21 (2005) 2281.

32. S. J. Sze and T. Y. Lee, Phys. Rev. B 51 (1995) 8709.

33. Y. B. Melnichenko, G.. D. Wignall, D. R. Cole, and H. Frielinghaus, J. Chem. Phys. 124

(2006) 204711.

34. Y. B. Melnichenko, G.. D. Wignall, Phys. Rev. E 69 (2004) 057102. 


\section{Figure captions}

Fig. 1 (a) - (c) Typical SEM images (left) and their two-digit images (right) at different magnification of fractal porous silica prepared at $r_{\mathrm{c}}=1$ and calcination temperature $T_{\text {cal }}=650$ ${ }^{\circ} \mathrm{C}$, where the black parts are real sectional parts and the white parts are pores. (d) Numerical results of box-counting method. The arrows show inflection points on the slope.

Fig. 2 (a) - (c) Typical SEM images at different magnification of fractal porous silica prepared at $r_{\mathrm{c}}=2$ and $T_{\text {cal }}=650{ }^{\circ} \mathrm{C}$. (d) Numerical results of box-counting method. The arrows show inflection points on the slope.

Fig. 3 (a) - (c) Typical SEM images at different magnification of fractal porous silica prepared at $r_{\mathrm{c}}=3$ and $T_{\text {cal }}=650{ }^{\circ} \mathrm{C}$. (d) Numerical results of box-counting method. The arrows show inflection points on the slope.

Fig. 4 (a) Dependences of cross-sectional fractal dimension at different $r_{\mathrm{c}}$ on calcination temperature of the fractal porous silicas. (b) Dependences of upper cutoff $L$ and lower one $l$ with different $r_{\mathrm{c}}$ on calcination temperature. The open and closed symbols are corresponding to $L$ and $l$, respectively. Squares, circles, and triangles denote the data at $r_{\mathrm{c}}=1,2$, and 3, respectively. 
Fig. 5 Pore size distribution obtained by mercury porosimetry of fractal porous silica samples at $r_{\mathrm{c}}=1(\mathrm{a}), 2$ (b), and 3 (c), respectively, and normal silica (d). $T_{\text {cal }}$ was $650{ }^{\circ} \mathrm{C}$.

Fig. 6 Dependences of total pore volume $V_{\mathrm{Hg}}$ (a) and porosity (b) of the fractal porous silicas at each $r_{\mathrm{c}}$ on $T_{\text {cal. }}$. The cumulative pore volume of the normal $\mathrm{SiO}_{2}$ is also shown in (a).

Fig. 7 Pore size distribution obtained from ${ }^{1} \mathrm{H}$ NMR experiment of fractal porous silica prepared at $r_{\mathrm{c}}=1$ and $T_{\text {cal }}=650{ }^{\circ} \mathrm{C}$. (a) Dependence of cumulative pore volume on pore size. (b) Pore size distributions obtained from $d V / d(\log r)$.

Fig. 8 Dependences of cumulative pore volume on pore size obtained by nitrogen gas adsorption. (a) - (c) are corresponding to the samples at $r_{\mathrm{c}}=1,2$, and 3, respectively. (d) Dependence of specific surface area of samples with different $r_{\mathrm{c}}$ on calcinations temperature.

Fig. 9 Pore size distributions from $r=1 \mathrm{~nm}$ to $10 \mu \mathrm{m}$; the data are exhibited all together obtained from the mercury porosimetry (obtained from Fig. 5 (a)), the ${ }^{1} \mathrm{H}$ NMR cryoporometric experiment (Fig. 7 (b)), and the nitrogen gas adsorption technique (obtained from the cumulative pore volume of $T_{\text {cal }}=650{ }^{\circ} \mathrm{C}$ in Fig. 8 (a)). 
Fig. 10 A scheme of ideal fractal porous silica assembled as Menger sponges. (a) The first, (b) the second, and (c) the third generation. (d) A fractal body assembled as Menger sponges.

Fig. 11 Comparison between the experimental and theoretical results on relative pore volume (right vertical axis) and cumulative pore volume (left vertical axis) obtained from mercury porosimetry. The experimental results is the fractal porous silica prepared at $r_{\mathrm{c}}=1$ and $T_{\text {cal }}=$ $650{ }^{\circ} \mathrm{C}$. The solid line is the obtained cumulative pore volume on $r$ and the low histogram is the relative pore volume in Fig. 5 (a). The large histogram is obtained by a classification of the low histogram for the comparison with the theoretical one. Open triangles show theoretical dependences of cumulative pore volume obtained from Menger sponge. Filled triangles indicate theoretical dependences of relative pore volume. The maximum pore size $L$ is assumed to be 1.3 $\mu \mathrm{m}$.

Fig. 12 (a) A schematic representation of a fractal sponge model, where $D_{\mathrm{cs}}=-\log 13 / \log (1 / 4)=$ 1.85 and $D=-\log 39 / \log (1 / 4)=2.64$. (b) Comparison between the experimental and theoretical results on relative pore volume (right vertical axis) and cumulative pore volume (left vertical axis) obtained from mercury porosimetry. The experimental results is the fractal porous silica prepared at $r_{\mathrm{c}}=2$ and $T_{\text {cal }}=650{ }^{\circ} \mathrm{C}$. The solid line is the obtained cumulative pore volume on $r$ and the low histogram is the relative pore volume in Fig. 5 (b). 
Fig. 13 (a) A schematic representation of a fractal sponge model, where $D_{\mathrm{cs}}=-\log 12 / \log (1 / 4)=$ 1.79 and $D=-\log 32 / \log (1 / 4)=2.50$. (b) Comparison between the experimental and theoretical results on relative pore volume (right vertical axis) and cumulative pore volume (left vertical axis) obtained from mercury porosimetry. The experimental results is the fractal porous silica prepared at $r_{\mathrm{c}}=3$ and $T_{\text {cal }}=650{ }^{\circ} \mathrm{C}$. The solid line is the obtained cumulative pore volume on $r$ and the low histogram is the relative pore volume in Fig. 5 (c).

Fig. 14 (a) Sierpinsly carpet (equal to the cross section of Menger sponge) and (b) a fractal sponge model (equal to the cross section of the model in Fig. 13 (a)), where the $L$ is fixed and their $D_{\mathrm{cs}}$ are shown. 
(a)
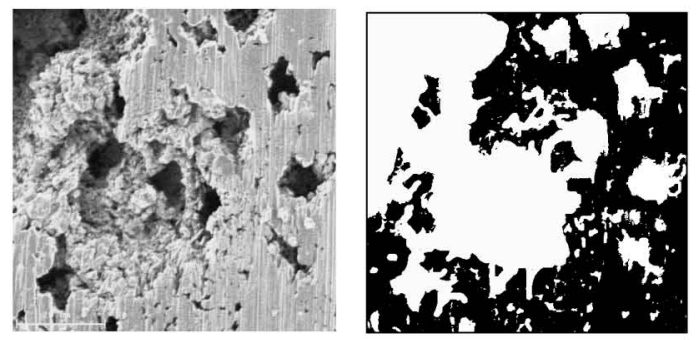

$20 \mu \mathrm{m}$

(b)
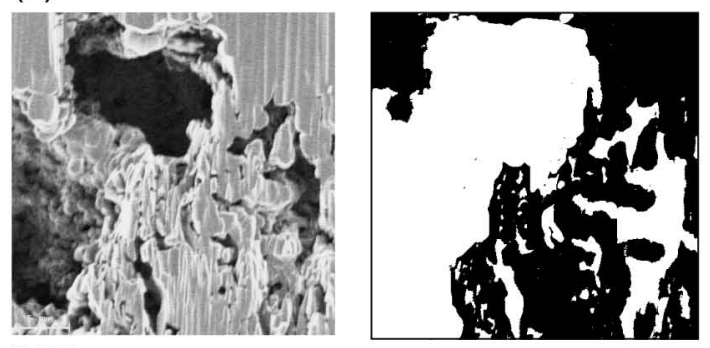

$5 \mu \mathrm{m}$

(c)
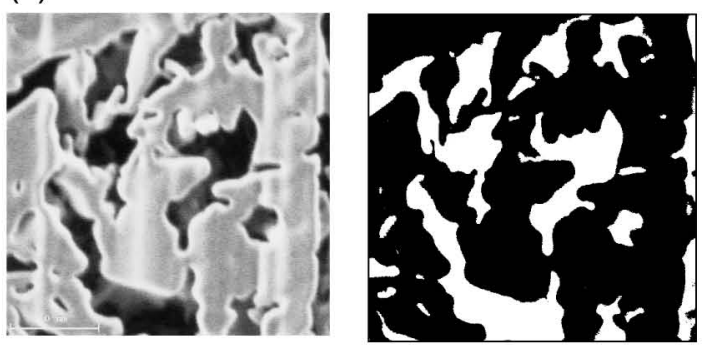

$1.0 \mu \mathrm{m}$

(d)

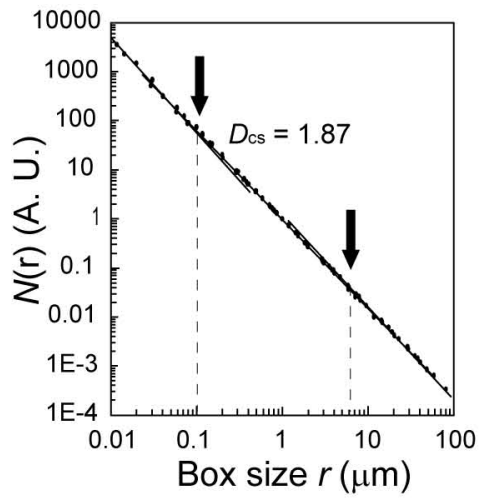

Fig. 1 Ono et al. 


\section{(a)}
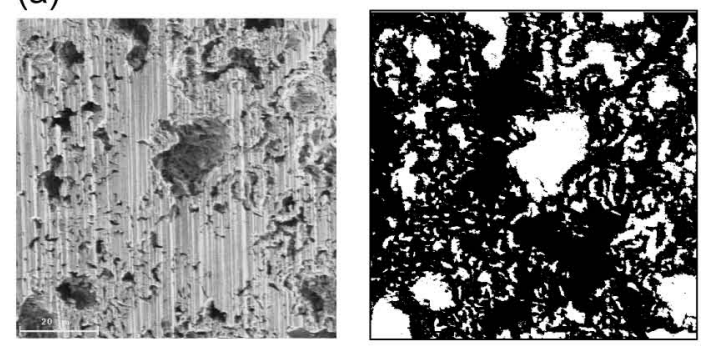

$20 \mu \mathrm{m}$

(b)
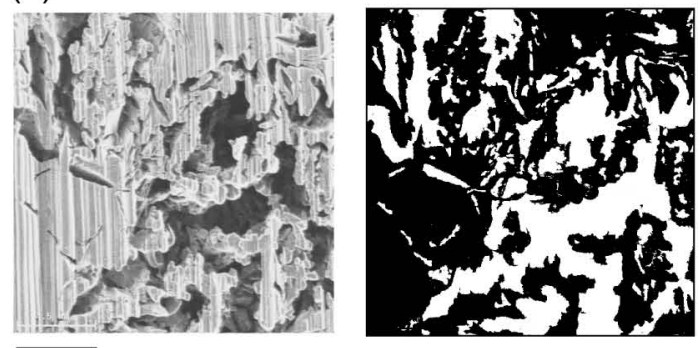

$4.5 \mu \mathrm{m}$

(c)
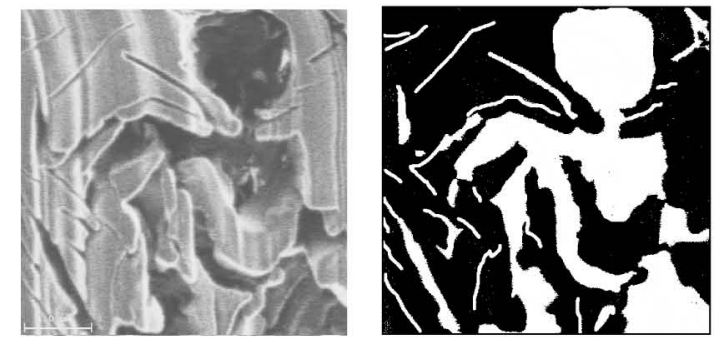

$\overline{1.0 \mu \mathrm{m}}$

(d)

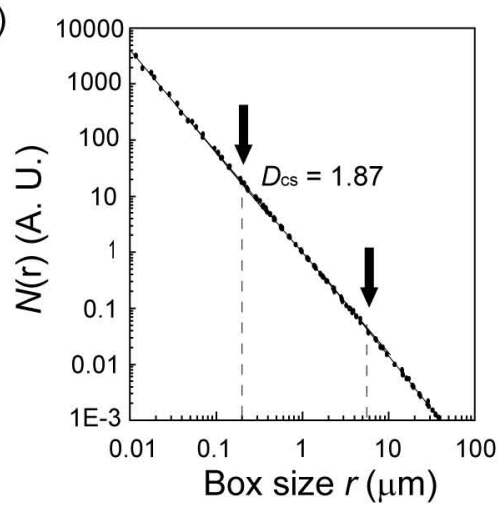

Fig. 2 Ono et al. 
(a)
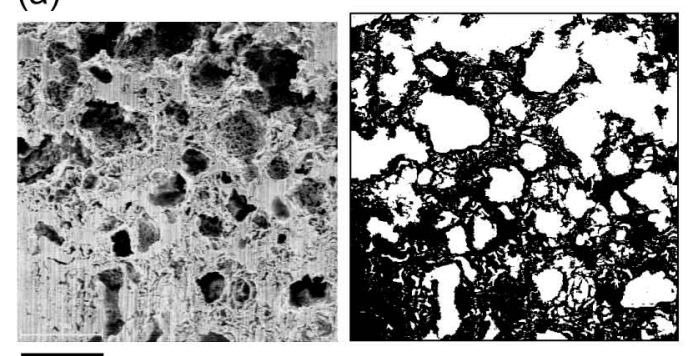

$25 \mu \mathrm{m}$

(b)

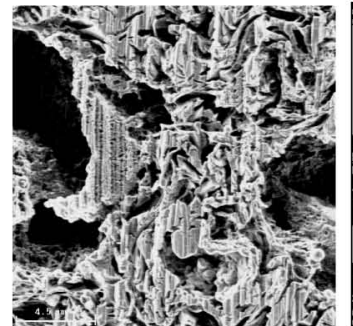

atolving

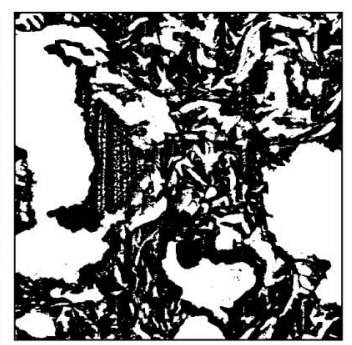

$4.5 \mu \mathrm{m}$

(c)
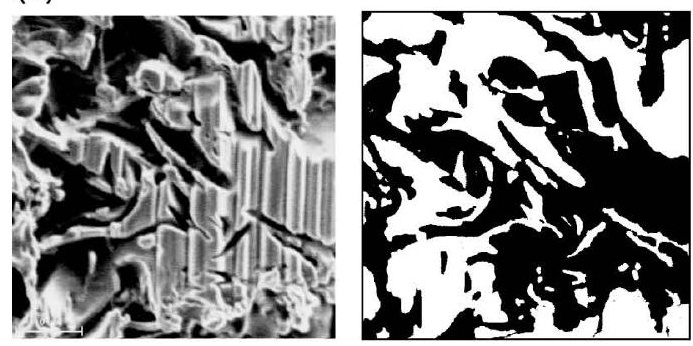

$1.0 \mu \mathrm{m}$

(d)

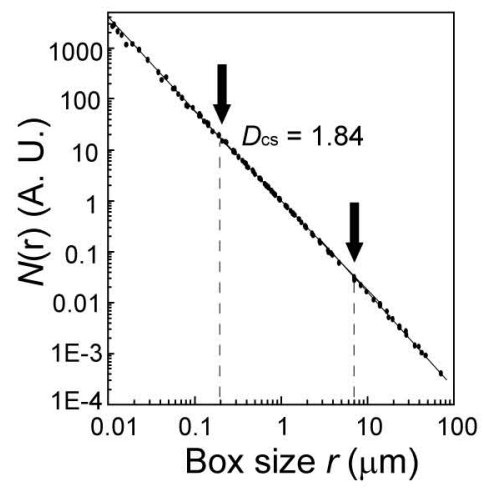

Fig. 3 Ono et al. 


\section{(a)}

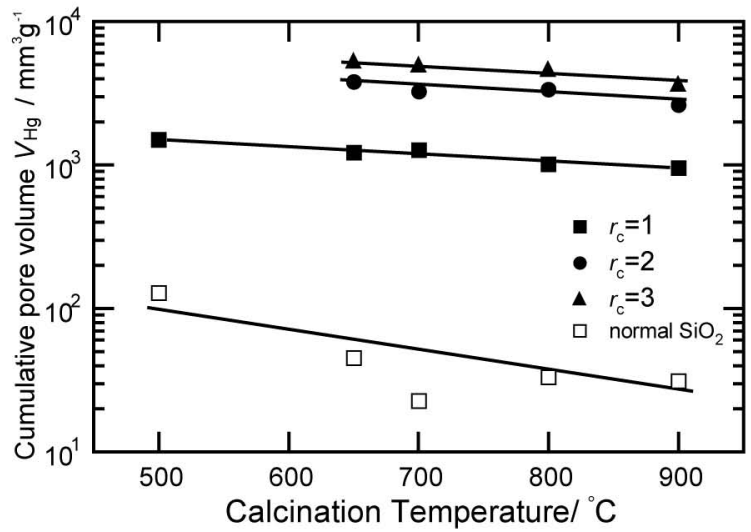

(b)

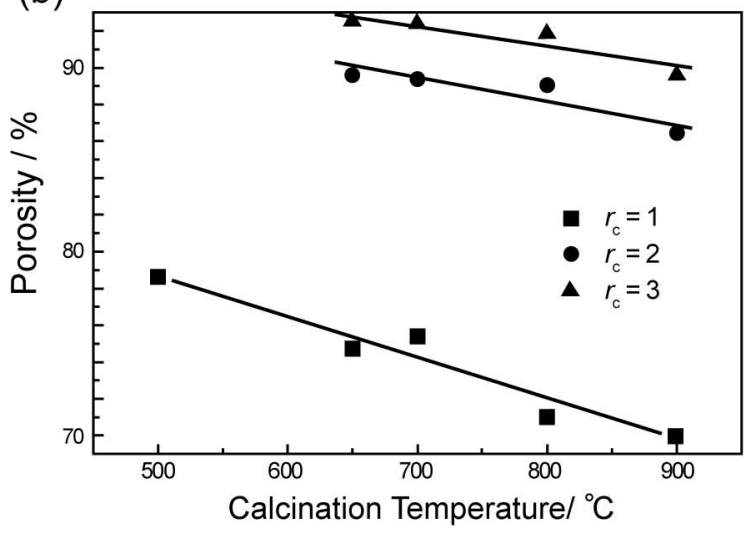

Fig. 6 Ono et al. 


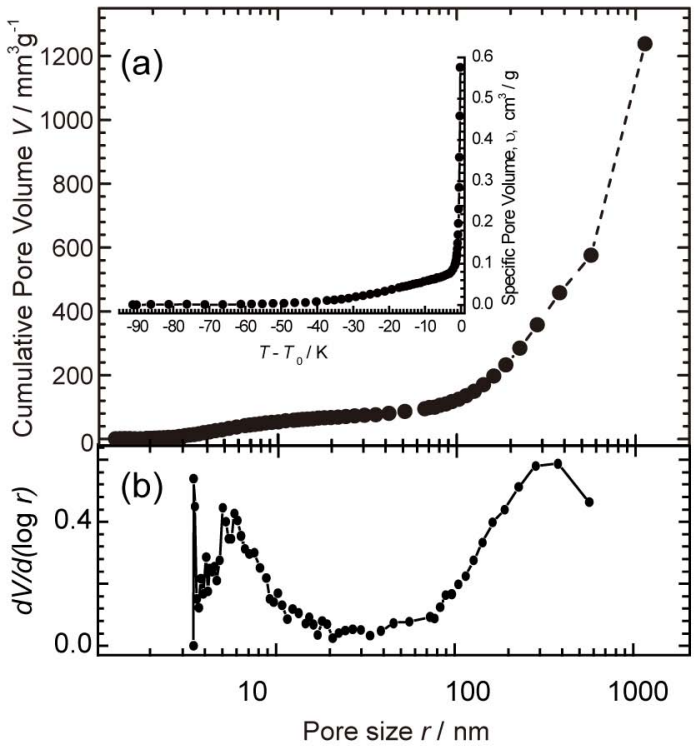




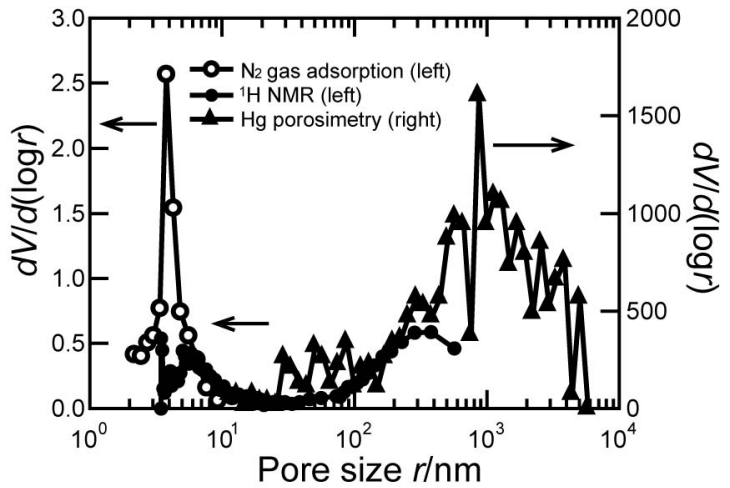


(a)

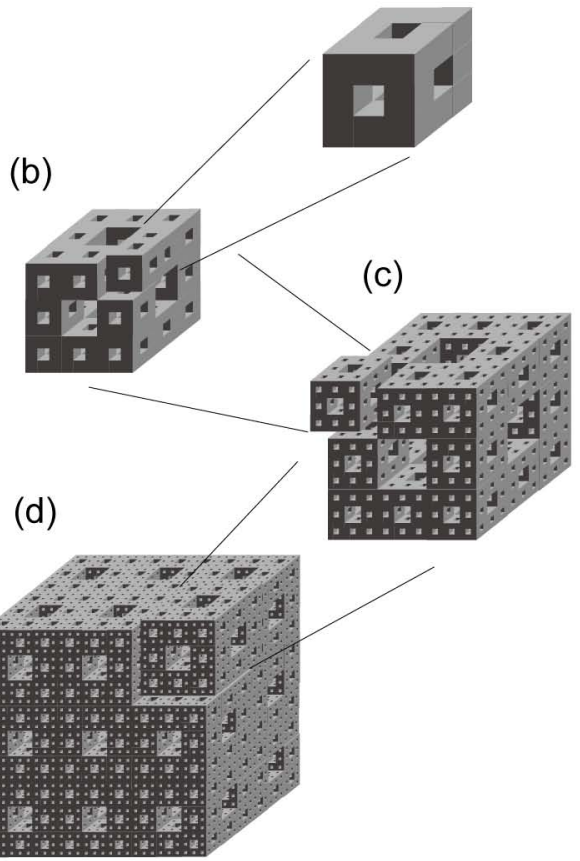

\section{Fig. 10 Ono et al.}




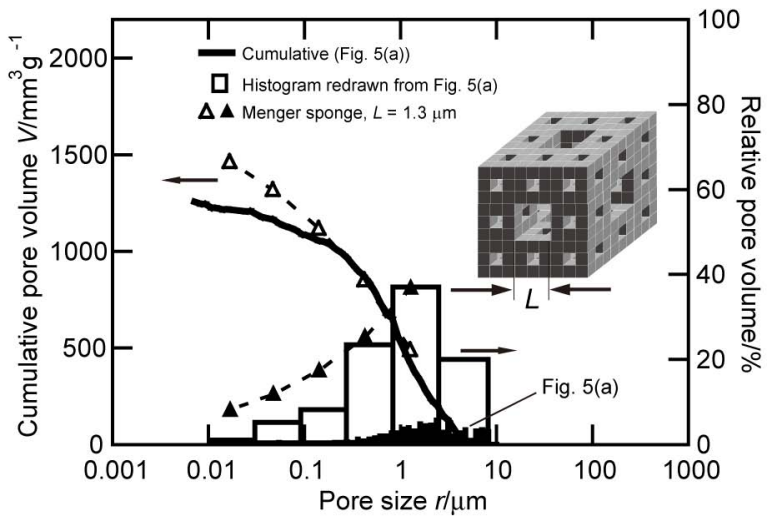

Fig. 11 Ono et al. 
(a)

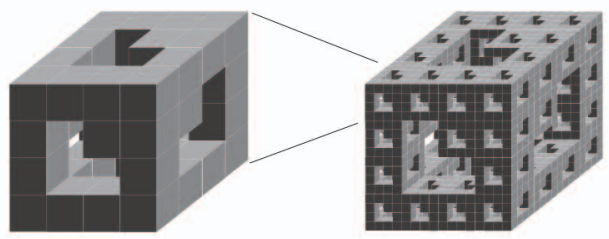

(b)

$$
\begin{aligned}
& D_{\mathrm{cs}}=-\log 13 / \log (1 / 4)=1.85 \\
& D=-\log 39 / \log (1 / 4)=2.64
\end{aligned}
$$

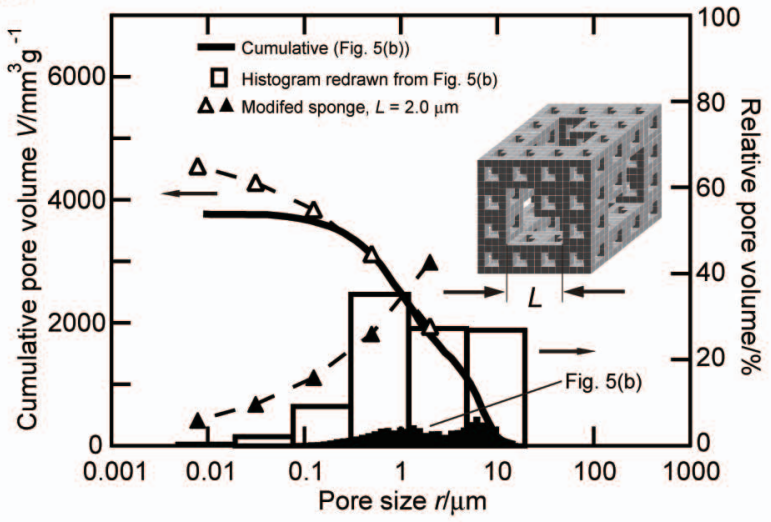

Fig. 12 Ono et al. 
(a)

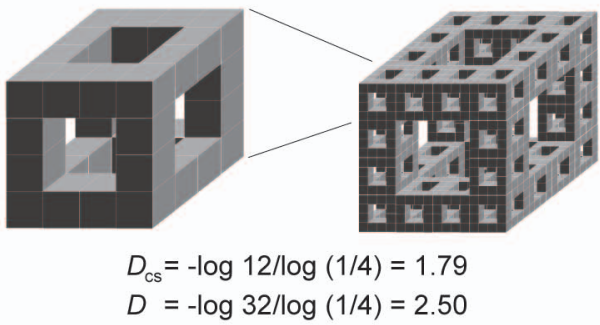

(b)

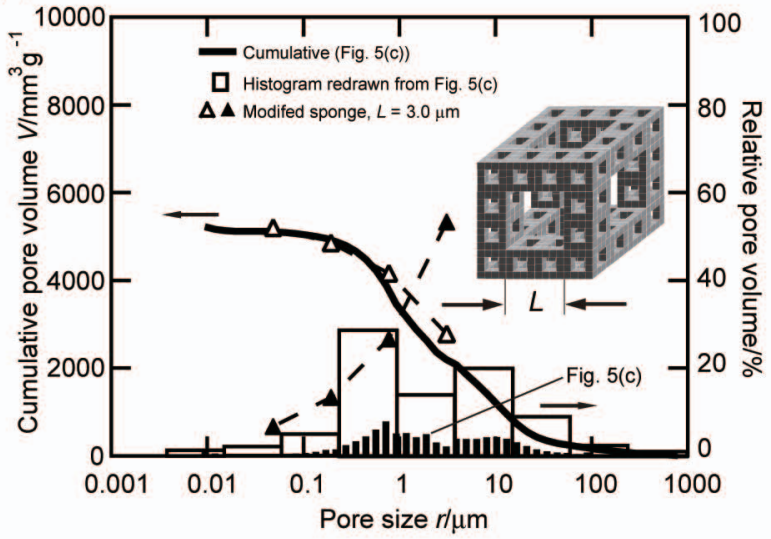

Fig. 13 Ono et al. 
(a)

(b)

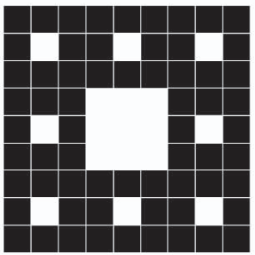

$D_{c s}=-\log 8 / \log (1 / 3)=1.89 \quad D_{c s}=-\log 12 / \log (1 / 4)=1.79$ 